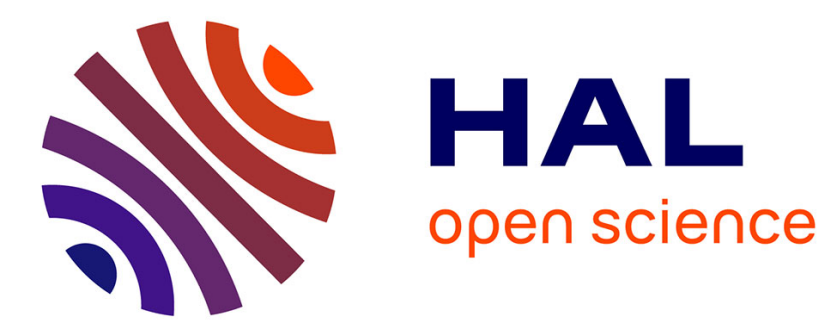

\title{
Traducteur ultrasonore à caustique cylindrique ou sphérique
}

\author{
J. Perdijon
}

\section{To cite this version:}

J. Perdijon. Traducteur ultrasonore à caustique cylindrique ou sphérique. Revue de Physique Appliquée, 1974, 9 (5), pp.925-929. 10.1051/rphysap:0197400905092500 . jpa-00243857

\section{HAL Id: jpa-00243857 https://hal.science/jpa-00243857}

Submitted on 1 Jan 1974

HAL is a multi-disciplinary open access archive for the deposit and dissemination of scientific research documents, whether they are published or not. The documents may come from teaching and research institutions in France or abroad, or from public or private research centers.
L'archive ouverte pluridisciplinaire HAL, est destinée au dépôt et à la diffusion de documents scientifiques de niveau recherche, publiés ou non, émanant des établissements d'enseignement et de recherche français ou étrangers, des laboratoires publics ou privés. 


\title{
TRADUCTEUR ULTRASONORE A CAUSTIQUE CYLINDRIQUE OU SPHÉRIQUE
}

\author{
J. PERDIJON \\ Centre d'Etudes Nucléaires, 38041 Grenoble, France
}

(Reçu le 18 mars 1974)

\begin{abstract}
Résumé. - Lors de certains contrôles par ultrasons, il est nécessaire de disposer de faisceaux dont tous les rayons frappent l'objet à contrôler sous la même incidence. Si la section de l'objet par le plan d'incidence est un cercle, la section des surfaces d'ondes incidentes par le même plan doit alors être une portion de développante de cercle. On a déterminé la forme de divers traducteurs produisant de telles surfaces; leur angle d'ouverture pouvant atteindre $360^{\circ}$, ils permettraient en particulier le contrôle des tubes minces sans mise en rotation.
\end{abstract}

\begin{abstract}
Ultrasonic beams all rays of which strike the object to be tested at the same angle would often be useful. If the section of the object in the plane of incidence is a circle, the section of each incident wave front must be a part of an involute of circle. We have determined the shape of some transducers which produce such wave fronts. As their aperture may reach $360^{\circ}$, it would be possible to examine thin-walled tubes without rotating them.
\end{abstract}

1. Introduction. - Lorsqu'on explore un objet en vue du contrôle de sa santé au moyen d'un faisceau ultrasonore, il faut pouvoir délimiter précisément la zone examinée afin de connaître la répartition de l'énergie à l'intérieur de l'objet et de pouvoir ainsi interpréter correctement les échos observés.

Cette délimitation peut se faire de deux façons :

- soit en utilisant un faisceau focalisé à l'intérieur de l'objet ; l'énergie est alors concentrée autour du foyer,

- soit en utilisant un faisceau dont tous les rayons font, après réfraction, le même angle avec la normale au dioptre milieu de couplage-objet ; l'énergie est alors concentrée sur l'enveloppe des rayons réfractés.

On connaît bien l'émission des faisceaux focalisés [1] et leur propagation dans le cas de divers dioptres. Lorsque les sections du dioptre par les plans d'incidence sont des segments de droite, il est, d'autre part, évident que les sections de la pastille piézoélectrique par les mêmes plans doivent être également des segments de droite pour que l'angle de réfraction de tous les rayons soit constant. Nous allons étudier quelle doit être la forme du traducteur pour obtenir cette même constance de l'angle de réfraction $r$ quand les sections du dioptre par les plans d'incidence sont des cercles de rayon $R$; en effet, un tel traducteur pourrait trouver de nombreuses applications dans le contrôle des objets cylindriques ou sphériques comme, par exemple :
- l'exploration d'un produit massif ou épais sur une profondeur $p$, en ondes longitudinales ou transversales (on a alors : $r=\operatorname{Arcsin}(1-p / R)$ ),

- le contrôle d'un produit mince en ondes de $\operatorname{Lamb}(r=\pi / 2)$.

2. Détermination de la forme des surfaces d'ondes incidentes. - Si on désire que l'angle de réfraction $r$ soit constant, l'angle d'incidence $i$ doit lui-même être constant. Puisque la section de l'objet par un plan d'incidence est un cercle $\mathrm{C}$, de centre $\mathrm{O}$ et de rayon $R$, tous les rayons situés dans ce plan ont une incidence $i$ constante si leurs prolongements sont tangents au cercle $\mathrm{C}^{\prime}$, de centre $\mathrm{O}$ et de rayon $a=R \sin i$.

Les surfaces d'ondes étant orthogonales aux rayons, leurs sections par le même plan d'incidence doivent donc être des développantes du cercle $C^{\prime}$. L'équation de ces développantes en fonction du paramètre $\varphi$ est (axes orthogonaux centrés en $O, k$ désignant une constante) :

$$
\left.\begin{array}{l}
x=a[\cos \varphi+(\varphi+k) \sin \varphi] \\
y=a[\sin \varphi-(\varphi+k) \cos \varphi]
\end{array}\right\} .
$$

Dans le cas d'un cylindre de révolution, les surfaces d'ondes sont obtenues par translation de ces développantes parallèlement à l'axe du cylindre. Dans le cas d'une sphère, elles sont obtenues par rotation des développantes autour d'un diamètre du cercle $\mathrm{C}$. 
3. Détermination de la forme du traducteur. - Pour obtenir de telles surfaces d'ondes, on peut soit donner une forme appropriée à la pastille piézoélectrique, soit accoler une lentille ou bien associer un miroir à une pastille plane (objet cylindrique) ou cylindrique de révolution (objet sphérique) [2].

Nous allons d'abord déterminer quelle doit être la forme du traducteur dans le cas où les plans d'incidence sont normaux à la pastille. Il suffit alors de calculer la section de la pastille courbe, ou de la lentille, ou du miroir, par l'un des plans d'incidence et on obtient les surfaces correspondantes, selon la forme de l'objet, par le même type de déplacement que pour les surfaces d'ondes.

3.1 Pastille Courbe. - Nous avons obtenu au paragraphe 2 l'équation d'une section des surfaces d'ondes. Si on n'utilise ni lentille, ni miroir, la pastille doit constituer l'une de ces surfaces d'ondes et on obtient l'équation de sa section en donnant à $k$ une valeur donnée dans l'équation (1).
On peut faire les remarques suivantes :

a) Si le traducteur est placé à l'extérieur de l'objet, on a $|\varphi+k|>\operatorname{cotg} i$; si le traducteur est placé à l'intérieur de l'objet, on a $|\varphi+k|<\operatorname{cotg} i$ et la pastille doit être extérieure au cercle $C^{\prime}(R$ désignant alors le rayon de l'alésage);

b) Si $\varphi>-k, i$ est mesuré à partir de la normale dans le sens inverse ; si $\varphi<-k$, $i$ est mesuré dans le sens direct. Il peut être bon d'utiliser deux traducteurs correspondant l'un à $\varphi>-k$ et l'autre à $\varphi<-k$ afin d'examiner l'objet sous deux incidences opposées ;

c) $x$ et $y$ étant toujours définis, on peut faire varier $\varphi$ dans un intervalle de $2 \pi$. Le traducteur peut donc entourer complètement l'objet à examiner, ce qui évite d'avoir à faire tourner celui-ci relativement au traducteur pour obtenir l'exploration totale de l'une de ses sections.

Nous avons tracé (Fig. 1) la section de la pastille correspondant à : $i=30^{\circ}, k=3 \pi / 2,0<\varphi<2 \pi$.

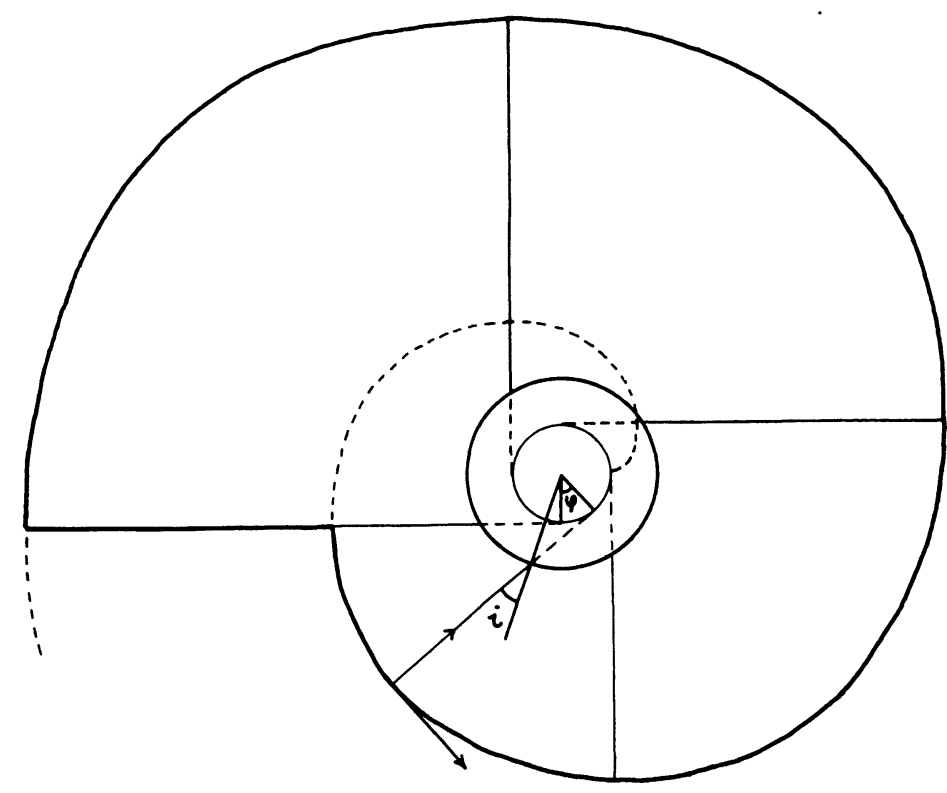

FIG. 1. - Pastille à caustique cylindrique ou sphérique.

3.2 Lentille. - En un point de la section de la lentille par l'un des plans d'incidence, côté milieu de couplage, on doit avoir (Fig. 2) :

- angle rayon incident-tangente à la section $=\alpha+\pi / 2$,

- rayon réfracté tangent au cercle $C^{\prime}$,

- $\sin \alpha=n \sin \beta$ ( $n$ désignant le rapport des vitesses de propagation dans la lentille et dans le milieu de couplage).

L'écriture de ces conditions permet d'obtenir l'équation recherchée (axes orthogonaux centrés en $\mathrm{O}, k$ désignant une constante) :

$$
\left.\begin{array}{l}
x=\frac{a n}{n-\cos \varphi}[-\sin \varphi+(\varphi+k) \cos \varphi] \\
y=\frac{a n}{n-\cos \varphi}\left[\cos \varphi-\frac{1}{n}+(\varphi+k) \sin \varphi\right]
\end{array}\right\} .
$$

On peut faire les remarques suivantes :

a) On peut choisir $k$ de façon que cette courbe soit extérieure à la section de l'objet ;

b) Pour obtenir une incidence opposée, il suffit de changer $y$ en $-y$;

c) L'interposition d'un milieu intermédiaire limite la portion utilisable de la courbe. Si $n>1$, la lentille est 


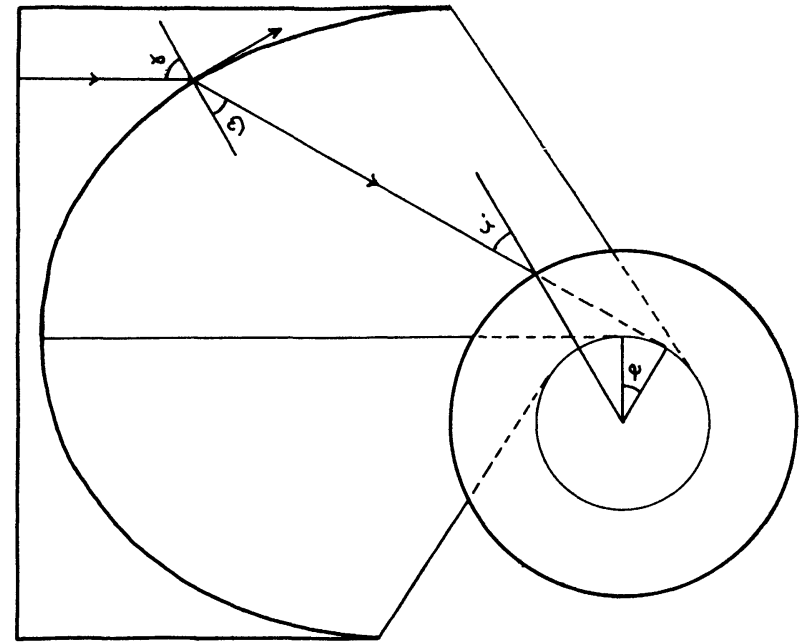

FIG. 2. - Pastille plane ou cylindrique accolée à une lentille à caustique cylindrique ou sphérique.

plan-concave et il faut $|\varphi|<\operatorname{Arc} \cos 1 / n$; si $n<1$, la lentille est plan-convexe, il faut $|\varphi|<\operatorname{Arc} \cos n$ et la courbe présente deux branches infinies.

Nous avons tracé (Fig. 2) la section de la lentille correspondant à : $i=30^{\circ}, n=1,8$ (lentille en araldite, eau comme milieu de couplage), $k=3,|\varphi|<56^{\circ}$.

3.3 Miroir. - En un point de la section du miroir par l'un des plans d'incidence, on doit avoir (Fig. 3) :

- angle rayon incident-tangente à la section $=\alpha+\pi / 2$,

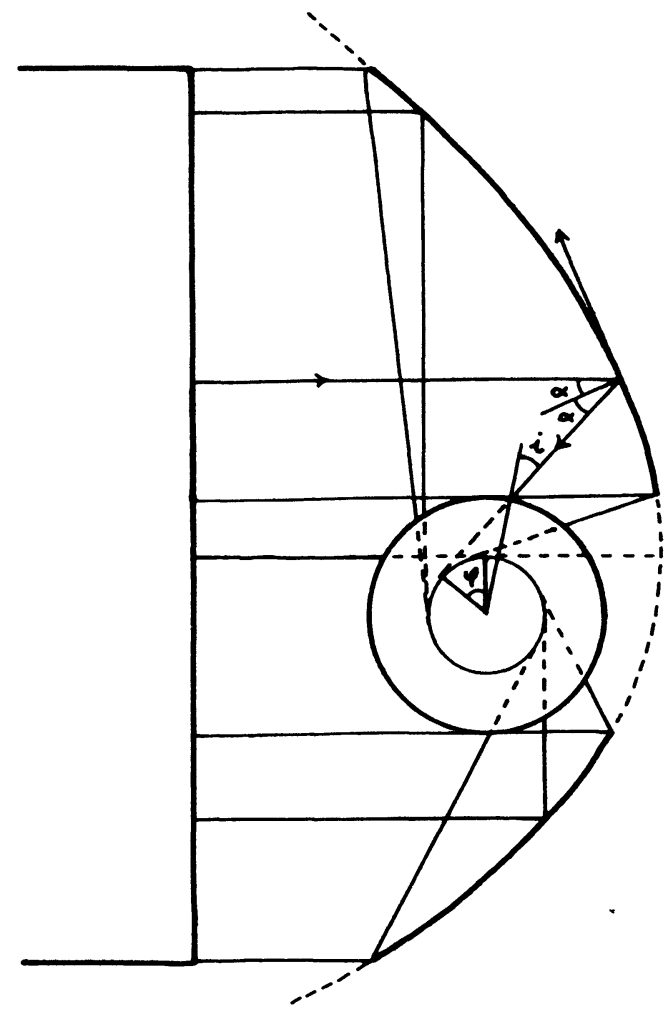

Frg. 3. - Pastille plane ou cylindrique associée à un miroir à caustique cylindrique ou sphérique.
- rayon réfléchi tangent au cercle $C^{\prime}$,

- angle rayon incident-rayon réfléchi $=2 \alpha$.

L'écriture de ces conditions permet d'obtenir l'équation recherchée (axes orthogonaux centrés en $\mathrm{O}$, $k$ désignant une constante) :

$$
\left.\begin{array}{l}
x=\frac{a}{1+\cos \varphi}[-\sin \varphi+(\varphi+k) \cos \varphi] \\
y=\frac{a}{1+\cos \varphi}[\cos \varphi+1+(\varphi+k) \sin \varphi]
\end{array}\right\} .
$$

On peut faire les remarques suivantes :

a) On peut choisir $k$ de façon que cette courbe soit extérieure à la section de l'objet ;

b) Pour obtenir une incidence opposée, il suffit de changer $y$ en $-y$;

c) On peut faire varier $\varphi$ dans un intervalle de $2 \pi$ mais l'ombre de l'objet et la présence de deux branches infinies limitent la portion utilisable de la courbe.

Nous avons tracé (Fig. 3) la section du miroir correspondant à : $i=30^{\circ}, k=6,|\varphi|<180^{\circ}$.

4. Miroir pour objet cylindrique. - On vient de voir (§ 3.3) que l'ombre de l'objet limitait l'intérêt d'un miroir. Pour supprimer cette ombre, on peut songer, dans le cas d'un objet cylindrique de révolution, à utiliser une pastille plane perpendiculaire à l'axe de l'objet ; ainsi les rayons sont émis parallèlement aux génératrices de l'objet [2].

En un point de la surface du miroir, on doit avoir (Fig. 4) :

- angle rayon incident-normale à la surface $=\pi / 4$,

- rayon réfléchi tangent au cylindre $\mathrm{D}$ de même axe que l'objet et de base le cercle $\mathbf{C}^{\prime}$.

L'écriture de ces conditions permet d'obtenir une surface réglée engendrée par une demi-droite intersection de deux plans d'équations (axes orthogonaux, $\mathrm{Oz}$ étant confondu avec l'axe de l'objet) :

$$
\left.\begin{array}{l}
x \cos \varphi+y \sin \varphi=a \\
z=-x \sin \varphi+y \cos \varphi+a \varphi
\end{array}\right\}
$$

avec $z>a \varphi$.

On peut faire les remarques suivantes :

a) Si le traducteur est placé à l'extérieur de l'objet, la pastille doit être du côté $z>0$; si le traducteur est placé à l'intérieur de l'objet, la pastille doit être du côté $z<0$ et le miroir doit être extérieur au cylindre D ;

b) Pour obtenir une incidence opposée, il suffit de changer $z$ en $-z$ dans l'équation du deuxième plan ;

c) $x, y$ et $z$ étant toujours définis, on peut faire varier $\varphi$ dans un intervalle de $2 \pi$ et le traducteur peut donc entourer complètement l'objet à examiner.

Nous avons représenté (Fig. 4) une perspective d'un miroir placé à l'extérieur de l'objet et correspondant à :

$$
i=30^{\circ} \quad 0<\varphi<2 \pi \text {. }
$$




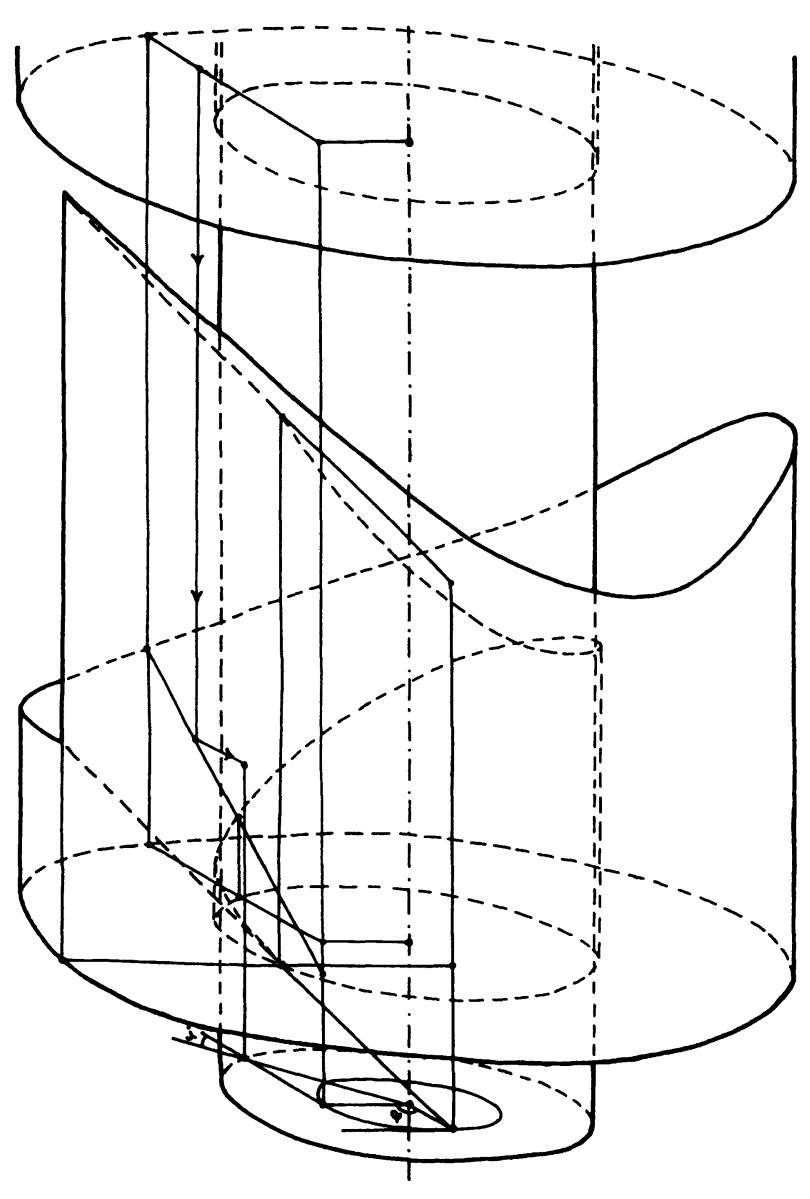

FIG. 4. - Pastille plane associée à un miroir à caustique cylindrique.

5. Application au contrôle des tubes minces. 5.1 Principe du Contrôle EN ONDES De LAMB. - Le contrôle de santé des tubes par ultrasons se fait actuellement par exploration hélicoïdale au moyen de deux traducteurs placés dans une cuve remplie d'eau [3] :

- l'un est situé dans un plan méridien du tube et son axe est incliné par rapport à la normale, de façon à détecter préférentiellement les défauts d'orientation perpendiculaire à l'axe du tube (défauts transversaux),

- l'autre est situé dans un plan équatorial mais son axe n'est pas concourant avec celui du tube, de façon à détecter préférentiellement les défauts d'orientation parallèle à l'axe du tube (défauts longitudinaux).

Dans le cas des tubes minces, le mode de propagation le mieux approprié est constitué par les ondes de Lamb [4] : elles se propagent parallèlement aux surfaces et toute l'épaisseur du matériau entre en vibration, ce qui permet une détection des défauts qui est indépendante de la profondeur à laquelle ceux-ci sont situés. Or, ces ondes ne peuvent être excitées dans un matériau donné, d'épaisseur donnée, et pour une fréquence donnée, que pour des angles d'incidence $i$ bien déterminés correspondant à chacun des modes symétriques et antisymétriques [5]. C'est ainsi que, pour un tube en acier inoxydable d'épaisseur $0,50 \mathrm{~mm}$ et pour une pastille piézoélectrique émettant des ultrasons de fréquence $4 \mathrm{MHz}$, l'angle d'incidence correspondant au mode Ao (le mieux adapté dans ce cas) est égal à $34^{\circ}$. Seuls les rayons frappant le tube sous cette incidence pourront engendrer des ondes de Lamb dans ce tube.

Pour que ces ondes soient suffisamment intenses, il existe une deuxième condition à remplir : les ondes de Lamb doivent rester en phase avec les ondes longitudinales du faisceau incident pendant au moins 4 ou 5 périodes. L'angle d'ouverture du faisceau, à l'intérieur duquel l'angle d'incidence est très voisin de l'angle correspondant à la génération des ondes de Lamb du mode choisi, doit donc être aussi grand que possible.

La réalisation de ces deux conditions ne pose pas de problème dans le cas de la détection des défauts transversaux : si la section de la pastille par un plan méridien d'incidence est un segment de droite, de longueur $d$ et faisant l'angle $i$ avec la surface du tube, les rayons émis dans ce plan ont tous une incidence $i$ et les ondes sont en phase sur une longueur de tube égale à $d / \cos i$ (Fig. 5). Par contre, ces conditions sont plus difficiles à obtenir pour les défauts longitudinaux.

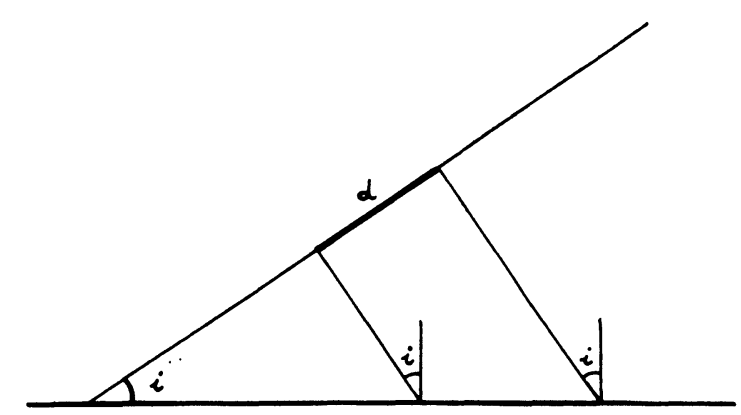

Fig. 5. - Détection des défauts transversaux.

5.2 DiffÉRENTS TRADUCTEURS POUR DÉFAUTS LONGITUDINAUX. - Les premiers traducteurs utilisés pour la détection des défauts longitudinaux dans les tubes étaient plans. On voit sur la figure 6 que, dans un plan équatorial d'incidence, seul un rayon a l'incidence $i$ correcte et l'arc de circonférence pour lequel les ondes sont en phase est très court.

On utilise maintenant des traducteurs à focalisation

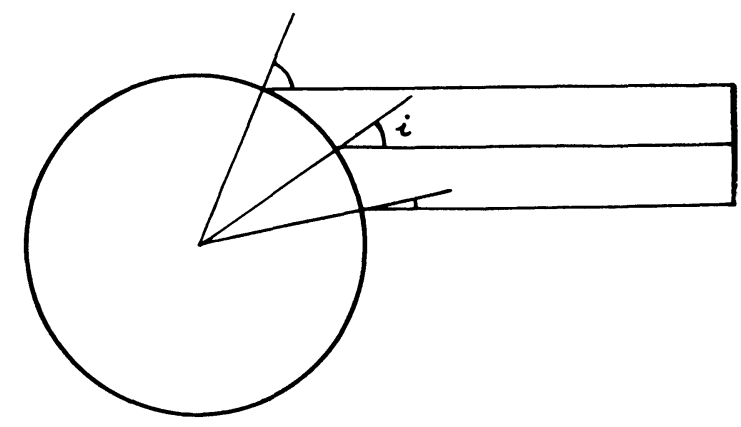

Fig. 6. - Détection des défauts longitudinaux avec un traducteur plan. 


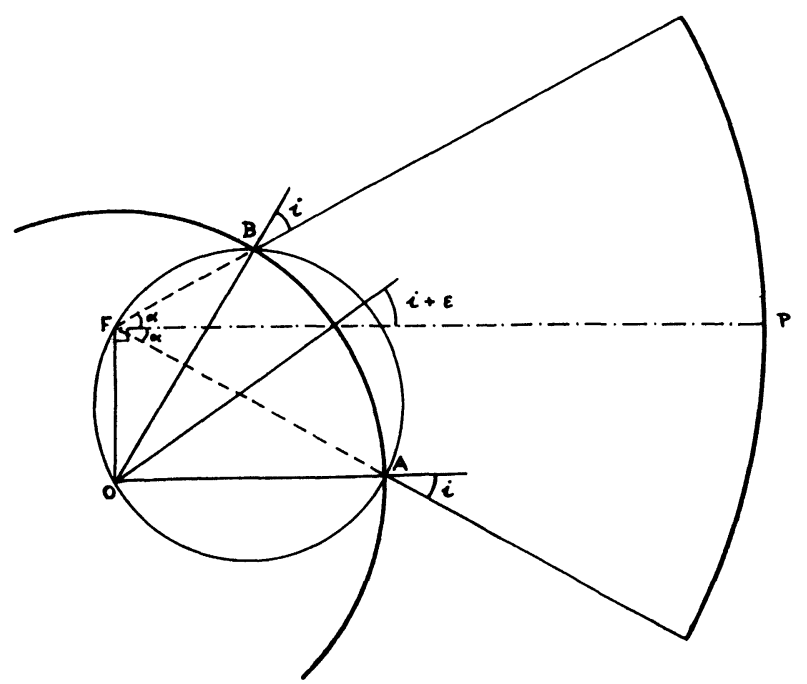

FIG. 7. - Détection des défauts longitudinaux avec un traducteur cylindrique.

cylindrique dont la ligne focale est orientée parallèlement à l'axe du tube [6]. On voit sur la figure 7 que, dans un plan équatorial d'incidence, le foyer $F$ doit être situé sur le cercle passant par le centre $O$ du tube et par les impacts $A$ et $B$ des rayons extrêmes pour que les incidences en A et en B soient égales. Cependant, l'inci- dence n'est pas constante à l'intérieur de l'arc $\overparen{\mathrm{AB}}$ et, plus celui-ci est long pour respecter la deuxième condition, plus la variation de l'incidence est importante.

5.3 UTILISATION D'UN TRADUCTEUR A CAUSTIQUE CYLINDRIQUE. - Avec un traducteur à caustique cylindrique, tous les rayons frappent le tube sous la même incidence $i$. La première condition est donc parfaitement remplie, sous réserve d'adapter la pastille, ou la lentille, ou le miroir, au rayon $R$ du tube et à l'angle $i$ imposé par la matière et l'épaisseur du tube, la fréquence de la pastille et le mode d'ondes de Lamb choisi.

De plus, il est possible d'obtenir cette constance de l'incidence sur un arc de circonférence très important qui peut même atteindre $360^{\circ}$ avec une pastille courbe (voir $\S 3.1$ et Fig. 1) ou avec un miroir réglé placé intérieurement ou extérieurement au tube (voir $\S 4$ et Fig. 4). La deuxième condition est donc remplie au point qu'on peut envisager la suppression de la mise en rotation du tube par rapport au traducteur. Il en résulterait des machines de défilement plus simples (une translation du tube ou du traducteur suffit) et un temps de contrôle beaucoup plus bref. A noter que, la hauteur d'eau étant variable, on ne serait plus gêné par la présence d'un écho d'entrée dans le tube.

\section{Bibliographie}

[1] Rozenberg, L. D. in Rozenberg, L. D., ed., Sources of high intensity ultrasound (Plenum Press, New York) 1969, vol. 1 , p. 223.

[2] PerdiJon, J., demande de brevet français EN 7346384 du 26 décembre 1973.
[3] Prot, A. et Monnier, P., rapport C. E. A. R 2577 (1964).

[4] VikTorov, I. A., Sov. Phys.-Acoust. 11 (1965) 1.

[5] Worlton, D. C., Hanford report H W 60662 (1959).

[6] Hanstock, R. F., Lumb, R. F., WALKeR, D. C. B., Ultrasonics 2 (1964) 109. 